\title{
When the drugs don't work
}

\author{
The identification of plasmid-borne resistance to an antibiotic of last resort suggests that the final \\ pharmacological barrier holding Gram-negative bacterial infections at bay may soon be breached.
}

Headlines proclaiming an antibiotic apocalypse and entry into a post-antibiotic era have become common place in recent years. While the use of such rhetoric risks desensitizing the wider public to the very real healthcare and economic problems associated with the spread of resistance, they have at the very least captured the attention of policy makers around the world. Efforts to address the limited pipeline of new antibiotic drugs being developed and to overcome resistance to drugs that are currently effective and approved for use in humans are increasing, but have we left it too late to close the gap? What will happen when the drugs of last resort are blunted and we are left with no therapeutic options? A recently published study (Y.-Y. Liu et al., Lancet Infect. Dis. http://doi.org/987; 2015) identifying $m c r-1$, a gene that confers resistance to colistin (a drug of last resort used to treat infections with Gram negative bacteria) suggests we may be about to find out.

Drugs of last resort represent the final pharmaceutical option that physicians fall back on when all other options have been exhausted. In the case of antibiotics, they are employed when infections prove resistant to the more commonly used drugs, whether in monotherapy or in combination. Last resort drugs should ideally be highly restricted both to limit the opportunity to select for resistance and because they sometimes have undesirable toxicity profiles and can cause serious side effects. For treating infections by Grampositive bacteria, the range of options for drugs of last resort while not particularly wide is at least viable in the near term, even for those strains that are resistant to the first line antibiotics. However, for Gram-negative bacteria the situation is critical. Plasmid-borne carbapenemases such as KPC, NDM-1 and VIM have spread widely among Enterobacteriaceae, Pseudomonas aeruginosa and Acinetobacter baumannii, conferring resistance to virtually all beta-lactams. Indeed, infections with Klebsiella pneumoniae carrying plasmids encoding VIM or NDM-1 genes are generally resistant to all drugs other than tigecycline and colistin, making for a rather thin last line of defence.
Into this setting steps $m c r-1$, which was recently identified in Escherichia coli strain SHP45 isolated from a pig in China, and found to confer resistance to colistin. Also known as polymyxin $\mathrm{E}$, colistin is a cationic polypeptide that exhibits broad spectrum activity against Gram-negative bacteria. Although developed in the 1950s, for a long period it was rarely used in treating humans as it had been linked with kidney damage. Resistance to colistin is not new; numerous bacterial species are intrinsically resistant while resistance has been shown to evolve in previously susceptible strains via chromosomal mutations. Colistin resistance for the most part relies on mutations that result in the modification of the lipid A moiety of lipopolysaccharide with phosphoethanolamine or 4-amino4-arabinose, which reduce the affinity for polymyxins. Indeed, the newly identified $m c r-1$ gene encodes a member of the phosphoethanolamine transferase family and catalyses the addition of phosphoethanolamine to lipid A. However, mcr- 1 differs to previous colistin resistance determinants in that it is borne on a plasmid, which enabled resistance to readily spread from SHP45 to another strain of E. coli. The $m c r-1$ plasmid could negate the therapeutic benefit of colistin use in a murine thigh model for E. coli infection and could also be maintained in $K$. pneumoniae and P. aeruginosa. Perhaps the most serious observation is that $\mathrm{mcr}-1$ carriage was observed in $15 \%$ of E. coli isolates taken from raw meat and $21 \%$ from animals in China during 2011-2014 and has subsequently been identified in isolates from other countries in Asia, Africa, Europe and North America - it is now effectively ubiquitous.

How worried should we be about $m c r-1$ ? Colistin use in humans varies by country; in most, colistin is employed at a relatively low frequency compared with other antibiotics (but is still in daily use in most cities in the UK, for example), whereas in other parts of the world colistin is effectively unrestricted. As such, when $m c r-1$ spreads into the clinic it will be a huge cause for concern. When $m c r-1$ spreads into strains that are already multi-drug resistant, pan-resistant infections that survive all treatment will increase as will the associated mortality and economic impact. This won't, however, mean that we have reached a so-called antibiotic apocalypse. Multi-drug resistance is generally associated with a fitness cost, so we might expect to face bacteria that are tougher to treat but have an impaired ability to cause infections. Furthermore, in much of the world there remains plenty of scope for improvement in the application of aseptic technique and infection control measures to limit the impact of $m c r-1$ carrying strains.

\section{In a globally connected society, there is no sense in reserving antibiotics for use as a last resort in some countries whilst other countries actively pump them into the environment through prophylactic use.}

Another area for which quick improvement can be made is in the immediate halt in the use of colistin in treating animals, whether as a growth promoter or therapeutically. For instance in China, where the $m c r-1$ gene was first identified, colistin is not currently used to treat humans but instead is added to animal feed as a growth promoter. Similarly, in many European countries prophylactic use of colistin in food animals is still regular practice. In a globally connected society, there is no sense in reserving antibiotics for use as a last resort in some countries whilst other countries actively pump them into the environment through prophylactic use. Resistance is mobile and does not respect international borders. Hopefully this situation will change in the coming months as efforts to persuade governments to ban agricultural colistin use are underway.

Ultimately, however, like all antibiotics, colistin will fail clinically as resistance spreads among pathogenic strains and becomes commonplace. There is still some time before this eventuality, but it will be important to use it wisely if we are to avoid the nightmarish headlines becoming a reality.

Corrected online: 1 February 2016 


\section{Correction}

In the Editorial 'When the drugs don't work' (Nature Microbiology 1, 16003; 2016), the reference in the first paragraph contained typographical errors and should have read: Y.-Y. Liu et al., Lancet Infect. Dis. http://doi. org/987; 2015. This has been corrected 1 February 2016. 